\title{
Neuropsychiatric Symptom Profile Differs Based on Pathology in Patients with Clinically Diagnosed Behavioral Variant Frontotemporal Dementia
}

\author{
Gabriel C. Léger Sarah J. Banks \\ Cleveland Clinic Lou Ruvo Center for Brain Health, Las Vegas, Nev., USA
}

\section{Key Words}

Behavioral variant frontotemporal dementia - Neuropsychiatric Inventory Questionnaire · Neuropathology · National Alzheimer's Coordinating Center · Behavior-neuropathological correlation

\begin{abstract}
Background: Behavioral variant frontotemporal dementia (bvFTD) is pathologically heterogeneous. With emerging therapeutics, determining underlying pathology during life is increasingly important. Neuropsychiatric symptoms are prevalent and diagnostic in bvFTD. Methods: We assessed the neuropsychiatric profile of patients with clinically diagnosed bvFTD as a function of pathology at autopsy. Patients with a clinical diagnosis of bvFTD at the initial visit were selected from the National Alzheimer's Coordinating Center (NACC) database. Neuropsychiatric symptoms endorsed on the Neuropsychiatric Inventory Questionnaire (NPI-Q) were analyzed. Results: Of 149 patients with clinically diagnosed bvFTD, pathology was primarily Alzheimer's disease (AD) in $20.5 \%$. These patients differed from those with underlying frontotemporal lobar degeneration: patients with AD pathology (plaques and tangles) were more likely to have hallucinations, delusions, or agitation. Patients were further differentiated into tau-positive (30\% of cases, including Pick's disease, FTD and parkinsonism with tau-positive or argyrophilic inclusions, and other tauopathies) or tau-negative cases (70\% of cases, including bvFTD tau-negative ubiquitin-positive inclusions). These patients also differed in some of the neuropsychiatric symptoms seen. Tau-negative cases were more likely to demonstrate depression, delusions, and changes in appetite and eating. Conclusions: These preliminary findings contribute to our increasing ability to predict, using simple clinical tools, the neuropathological underpinnings of bVFTD during life.




\section{Introduction}

Frontotemporal dementia (FTD) - the preferred term now being promoted is frontotemporal degeneration (www.theaFTD.org) - is the clinical manifestation of a number of distinct neurodegenerative processes collectively labeled frontotemporal lobar degeneration (FTLD) [1]. With recent advancements in molecular staining, the different neuropathological underpinnings of these processes are being described [2]. Broadly, FTLD can be subdivided into pathology that is either tau positive (approx. 50\%) or tau negative [3]. In addition to this pathological heterogeneity, FTLD can present as any of a number of distinct clinical profiles, including the behavioral variant FTD (bvFTD), the language variants (semantic and agrammatic) [4], and finally as parkinsonian or motor neuron disease syndromes [5].

As is the case in Alzheimer's disease (AD), there are currently no available therapeutics that target the underlying pathological mechanisms (amyloid plaques and tau-related tangles - henceforth referred to as pathological AD). Drugs targeting the pathological process are under development [6], but their efficacy (and our ability to demonstrate it) will depend on the selection of patients with a specific pathology, a process that remains challenging during life, even in the best academic centers. This diagnostic specificity will require two major distinctions: a correct diagnosis of bvFTD, and then the prediction of the underlying pathology. bvFTD is commonly misdiagnosed as AD, and, particularly in the absence of a genetic mutation, the prediction of its pathology is currently limited.

The diagnostic criteria for bvFTD require the presence of behavioral changes, including (1) disinhibition, (2) apathy, (3) loss of empathy, (4) new motor behaviors that are either compulsive, perseverative, or stereotyped, and (5) dietary changes including hyperorality [5, 7]. Many scales exist that attempt to capture and quantitate such changes [8-15], some of which have been specifically designed based on those changes most prevalent in FTD. The Neuropsychiatric Inventory (NPI) [8] is a scale that assesses behavioral and neuropsychiatric changes according to 12 subgroups. The NPI has been used to help differentiate bvFTD from other dementias, including AD [16-18]. A brief validated caregiver questionnaire version of this inventory has also been developed and validated: the NPI Questionnaire (NPI-Q) [19]. While the NPI is an interview-based scale, often requiring more than 15 min to complete (making it impractical in a general clinical practice setting), the NPI-Q is a series of specific validated questions derived from the NPI that can be answered in only a few minutes by a questionnaire administered to the caregiver. Conclusions drawn from the NPI-Q have been found to differ by no more than 5\% from those of the NPI [19].

Comparisons of neuropsychiatric symptoms in patients with clinical diagnoses of dementia have demonstrated distinctions between clinically (but not pathologically) diagnosed AD and bvFTD. Bathgate et al. [14] explored this distinction using their own caregiver questionnaire. They found that changes in emotions and insight, selfishness, disinhibition, personal neglect, gluttony and sweet food preference, wandering, stereotypies, loss of sensitivity to pain, echolalia, and mutism were more characteristics of bvFTD and differentiated the most bvFTD cases from AD cases. Bozeat et al. [13] also designed their own questionnaire, based largely on the NPI. They demonstrated that only the presence of either stereotypic and eating behaviors or loss of social awareness reliably differentiated patients with clinically diagnosed bvFTD from patients with AD. Kertesz et al. [10,20] designed the Frontal Behavioral Inventory (FBI) to probe for the presence of particular behavioral elements that are known to predict FTLD. This scale is both sensitive and specific at differentiating FTD from $\mathrm{AD}$ and also appears sensitive to progression [21]. In a large, well-defined clinical cohort, it has also demonstrated a strong correlation with the changes captured by the NPI and was better able to differentiate AD from bvFTD [22]. 
Léger and Banks: Neuropsychiatric Symptom Profile Differs Based on Pathology in

Other studies with standard or specifically designed scales to assess behavioral changes or changes associated with frontal lobe pathology have been variably successful in differentiating AD from bvFTD $[10,11,15,16,18,23-25]$. In particular, Levy et al. [16] used the NPI to show that, compared to patients with $\mathrm{AD}$, patients with FTD were more likely to exhibit apathy, disinhibition, euphoria, and aberrant motor behaviors. Using a validated Japanese translation of the NPI, Hirono et al. [18] showed nearly identical distinctions demonstrating that patients with bvFTD had significantly more euphoria, aberrant motor activity, and disinhibition, but also significantly fewer delusions compared to patients with AD [18]. Nagahama et al. [25], using the Cambridge Behavioral Inventory (CBI) initially developed by Bozeat et al. [13], showed that disinhibition, stereotypic behavior, elation, anxiety, poor self-care, and changes in eating habits occurred more frequently in patients with bvFTD than in patients with AD. Mendez et al. [11] used the BEHAVE-AD scale to show that patients with bvFTD were more likely to have worse overall scores, with significantly higher incidences of verbal outbursts (inappropriate personal comments) and inappropriate activity (disinhibited acts or immodest behavior), than patients with AD.

While the majority of studies on neuropsychiatric symptoms involve clinically diagnosed patients, Liscic et al. [26] showed that behavioral abnormalities in general, including impulsivity, disinhibition, social withdrawal, hyperorality as well as aphasia, were more likely present in patients with pathologically confirmed FTLD than in patients with pathologically confirmed AD. Of note, nearly one quarter of the patients with FTLD were also found to have AD pathological changes.

While to date there is no published research using neuropsychiatric symptoms to separate tau-positive and tau-negative patients, other clinical changes have been investigated. In a large postmortem analysis of 114 cases from two major centers, Forman et al. [27] demonstrated an association of social and language difficulties and motor neuron disease with non-tau bvFTD pathologies, while bvFTD associated with tau was more likely to show parkinsonism. Detailed information on the neuropsychiatric changes was not available.

Specific neuropsychiatric symptoms have been previously associated with particular pathologies in dementias more generally. Perhaps most specific, hallucinations are considered to be related to underlying synucleinopathy in Lewy body disease [28], although more recently hallucinations have been described in progressive supranuclear palsy, a tauopathy [29]. In bvFTD patients with one particular genetic variant linked to chromosome 9 with taunegative pathology (and representing $8 \%$ of bvFTD patients in this recent study), this variant has been associated with particular symptoms such as psychosis at a much higher rate than in patients with bvFTD who do not have this genetic mutation, and a much lower rate of sweet food preference [30]. Otherwise, to date, there has been no specific set of symptoms associated with tau-negative or tau-positive pathology in bvFTD.

Because agents that target the underlying pathology are more likely to work when administered early in the course of the disease, securing an early accurate diagnosis is an important goal. Although certain clinical signs are more prevalent in association with a given underlying process - for instance, the presence of parkinsonism or motor neuron disease in a patient with clinically diagnosed bvFTD is suggestive of tau-positive or tau-negative pathology, respectively [31] -, such findings typically emerge later in the course of the illness. Advanced structural neuroimaging analysis can also increasingly predict the pathological status in groups of individuals [32], but this remains expensive, available only in select academic centers, and its reliability in individual patients is unproven. The identification of early clinical characteristics that betray the underlying pathological process would allow for a less expensive and more rapid orientation of patients with a particular pathology to appropriate clinical trials involving specific etiologic agents. Once agents have been isolated and proven effective, these early clinical characteristics would also help safely select future patients for a given agent. 
Léger and Banks: Neuropsychiatric Symptom Profile Differs Based on Pathology in

Patients with Clinically Diagnosed Behavioral Variant Frontotemporal Dementia

The National Alzheimer's Coordinating Center (NACC) database represents a unique opportunity to investigate in vivo symptomatology while knowing ultimate pathology derived at autopsy in a large sample. The database includes clinical details of patients diagnosed and followed longitudinally at academic AD centers across the country. The data consist of early and behaviorally (including the NPI-Q) well-characterized patients who have been followed to autopsy. We analyzed data from the first NPI-Q administered to patients with both clinically diagnosed $\mathrm{AD}$ and bvFTD, and explored for the existence of patterns of endorsement that could be associated with specific pathologies, including FTLD subtypes. While the use of inventories more sensitive to FTLD (such as the FBI) may have represented better choices, this analysis was constrained by its retrospective nature from an available data set and the initial decision by the creators of the database to include the NPI-Q.

\section{Methods}

\section{Participants}

Data from the NACC neuropathology data set were used. The NACC database contains data from 34 past and present AD centers. In our sample, locked March 2012, we included all cases of clinically diagnosed (initial visit) and autopsy-proven $\mathrm{AD}$ and all cases of clinically diagnosed bvFTD (initial visit) irrespective of pathological diagnosis. Details of these two groups of patients are presented in table 1.

We then used frequency statistics to compare the prevalence of each of the 12 NPI-Q symptoms in the two groups. The bvFTD group was further broken down into the type of ultimate pathology detected. Thirtyone $(20.5 \%)$ of the clinically diagnosed bvFTD cases had primary pathological diagnoses of AD. Eighty-two (54.3\%) of the cases had primary bvFTD diagnoses. Given that there are various tauopathies found in bvFTD, we divided the patients into tau-positive (including Pick's disease, FTD and parkinsonism with tau-positive or argyrophilic inclusions, and other tauopathies) and tau-negative cases (FTD tau-negative ubiquitinpositive inclusions). In the NACC data set, 18 of the bvFTD patients were not given specific pathologies, but rather 'other' histology was checked. In these cases, a note was written to explain the primary pathology. In 10 of these cases, the cause was clearly definable as an FTLD tauopathy (these had noted 'Pick's disease', 'PSP', 'FTD-P', or 'CBD') and a further 5 had a nontauopathy FTLD ('FTLD-U TDP', 'MND', or 'DLDH'). These cases were relabeled as FTLD tauopathy and FTLD nontauopathy, respectively. The demographic details of the bvFTD tauopathy and FTLD nontauopathy groups are shown in table 2. Details were not available on 39 cases.

\section{Results}

Comparison of Patients with a Clinical Diagnosis of bvFTD (Any Pathology) and

Pathologically Confirmed Cases of Clinically Diagnosed $A D$

Patients with clinically diagnosed bvFTD more frequently presented with symptoms of anxiety $\left(\chi^{2}=5.97, p=0.015\right)$, elation $\left(\chi^{2}=21.97, p<0.0005\right)$, apathy $\left(\chi^{2}=19.21, p<0.0005\right)$, disinhibition $\left(\chi^{2}=64.67, \mathrm{p}<0.0005\right)$, motor changes $\left(\chi^{2}=30.20, \mathrm{p}<0.0005\right)$, and nighttime behavior $\left(\chi^{2}=41.86, p<0.0005\right)$ compared to patients with pathologically confirmed clinical diagnoses of AD. These findings are depicted in figure 1.

Comparison of Clinically Diagnosed bvFTD Patients with AD versus FTLD Pathology

Of the patients diagnosed in clinic with bvFTD, those with AD pathology were significantly more often ascribed delusions $\left(\chi^{2}=7.36, p=0.007\right)$, hallucinations $\left(\chi^{2}=4.32, p=\right.$ $0.039)$, and agitation $\left(\chi^{2}=3.87, p=0.050\right)$ compared to those with one of the FTLD pathologies. These results are depicted in figure 2 . 
Table 1. Demographic details of tau-negative and tau-positive patients with clinically diagnosed bvFTD who had FTLD pathology

\begin{tabular}{l|l}
\hline \multicolumn{2}{l|}{ Dement Geriatr Cogn Disord 2014;37:104-112 } \\
\hline DOI: $\underline{10.1159 / 000354368}$ & $\begin{array}{l}\text { C } 2013 \text { S. Karger AG, Basel } \\
\text { www.karger.com/dem }\end{array}$ \\
\hline
\end{tabular}

Léger and Banks: Neuropsychiatric Symptom Profile Differs Based on Pathology in Patients with Clinically Diagnosed Behavioral Variant Frontotemporal Dementia

\begin{tabular}{lll}
\hline & $\begin{array}{l}\text { FTD tau negative } \\
(\mathrm{n}=77)\end{array}$ & $\begin{array}{l}\text { FTD tau positive } \\
(\mathrm{n}=33)\end{array}$ \\
\hline Age at death, years & $65.95(9.28)$ & $66.58(8.22)$ \\
Education, years & $15.64(3.26)$ & $14.91(2.35)$ \\
Male gender, \% & 68.8 & 63.6 \\
White race, \% & 93.5 & 97.0 \\
\hline
\end{tabular}

Values represent means (SD), unless otherwise indicated.

There were no differences in these factors between the two groups.
Table 2. Demographic details of the two clinical diagnostic groups

\begin{tabular}{lll}
\hline & FTD $(\mathrm{n}=149)$ & AD $(\mathrm{n}=578)$ \\
\hline Age at death, years & $67.15(9.30)$ & $79.98(10.84)^{*}$ \\
Education, years & $15.23(3.13)$ & $15.76(9.08)$ \\
Male gender, \% & $66.90^{*}$ & 56.8 \\
White race, \% & 95.40 & 94.4 \\
\hline
\end{tabular}

Values represent means (SD), unless otherwise indicated.

bvFTD patients were significantly younger than AD patients, and there were more men in the bvFTD group. ${ }^{*} \mathrm{p}<0.05$.

Comparison of Clinically Diagnosed bvFTD Patients with Tau-Negative versus Tau-Positive FTLD Pathology

Patients with bvFTD who had FTLD pathology were divided into tau-negative and taupositive cases. The patients with tau-negative pathology were more likely to show delusions $\left(\chi^{2}=6.41, p=0.012\right)$, depression $\left(\chi^{2}=7.46, p=0.007\right)$, and changes in appetite and eating $\left(\chi^{2}=5.27, p=0.022\right)$. These results are depicted in figure 3 .

\section{Discussion}

There is great interest in being able to define phenotypic signatures of the neuropathology underpinning clinical bvFTD during life, especially early in the course. Additionally, tools that predict pathology will be great aids in clinical diagnosis.

We examined the pattern of endorsement of items on the NPI-Q to identify possible links to the underlying pathology. We found that in patients with a clinical diagnosis of bvFTD but in whom the underlying pathology was actually $\mathrm{AD}$, the presence of delusions, hallucinations, and agitation was much more frequent. Endorsement patterns also differed in patients with pathological diagnoses of FTLD depending on the pathological subtype, with caregivers of patients with tau-negative pathology being more likely to endorse delusions, depression, and appetitive changes. To our knowledge, this is the first study that explores and isolates patterns of presenting neuropsychiatric changes as they relate to tau-negative or tau-positive pathological diagnosis in bvFTD.

Similar to previous studies $[13,14,16,18]$ that used patients without pathological verification of their clinical diagnosis, we found that patients with clinically diagnosed bvFTD had more apathy, elation, disinhibition, aberrant motor behaviors, nighttime behaviors, and changes in appetite and eating compared with pathologically confirmed patients with clini- 
Dementia

and Geriatric

Cognitive Disorders

Fig. 1. \% frequency symptom endorsement: bvFTD (clinical diagnosis, any pathological diagnosis) vs. AD (clinical diagnosis and pathological diagnosis). Significant difference: ${ }^{*} \mathrm{p}<0.05$ (see text for details).

Fig. 2. \% frequency symptom endorsement for bvFTD patients: FTD pathological diagnosis vs. AD pathological diagnosis. Significant difference: ${ }^{*} \mathrm{p}<0.05$ (see text for details).

Fig. 3. \% frequency symptom endorsement for bvFTD patients: tau-negative vs. tau-positive pathology. Significant difference: $* \mathrm{p}<0.05$ (see text for details).

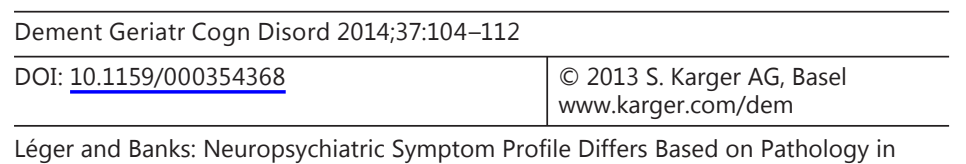

Léger and Banks: Neuropsychiatric Symptom Profile Differs Based on Pathology in Patients with Clinically Diagnosed Behavioral Variant Frontotemporal Dementia
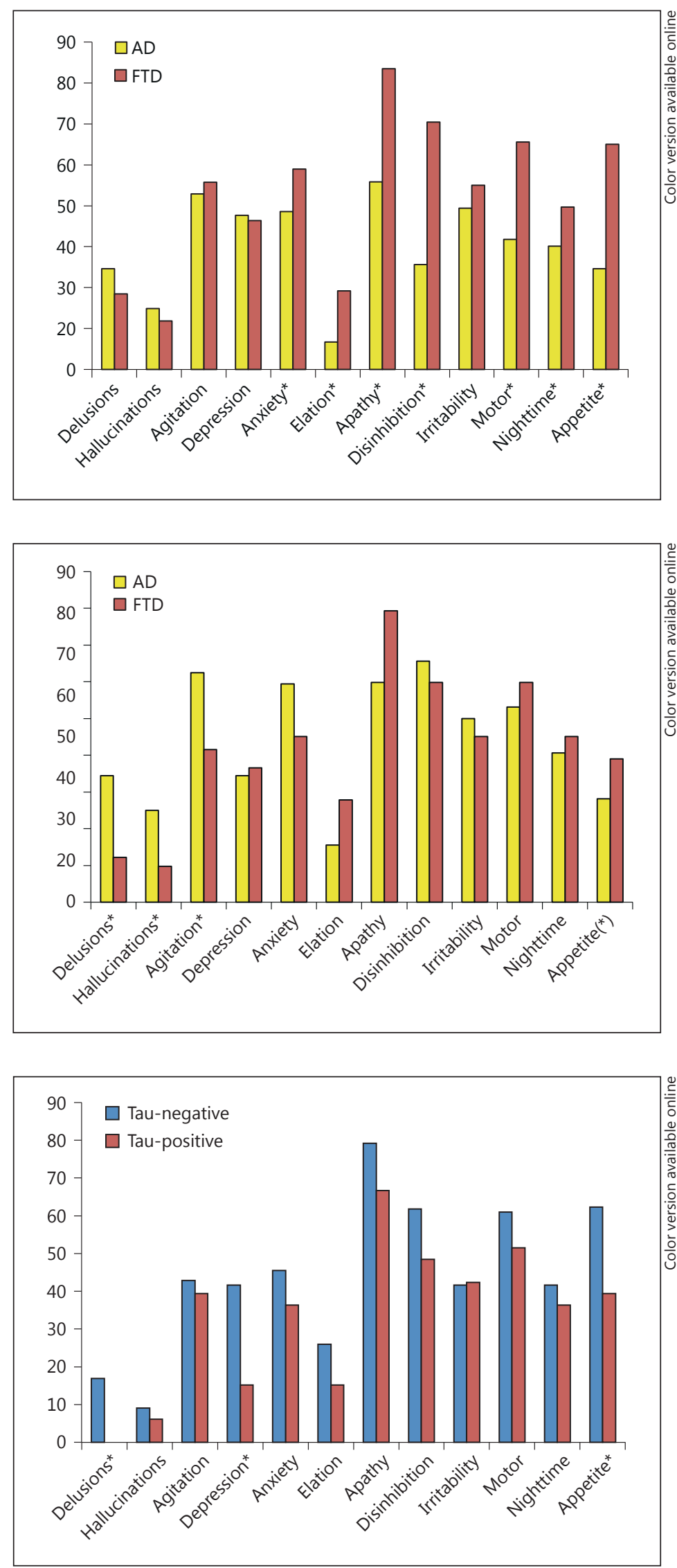
Léger and Banks: Neuropsychiatric Symptom Profile Differs Based on Pathology in

cally diagnosed $\mathrm{AD}$. This is not surprising given that criteria defining bvFTD require inclusion of a certain number of these neuropsychiatric symptoms for diagnosis. We further found that delusions, hallucinations, and agitation were more common in patients misdiagnosed clinically with bvFTD who had underlying AD pathology, compared to those with true FTLD pathology. This suggests that, for the most part, patients with psychoses go misdiagnosed. However, psychotic symptoms can occur in the minority of true FTLD cases [30, 33]. While we do not know what symptom profile led physicians in the NACC sites to define each of the patients, it could be that the clinically diagnosed bvFTD patients with AD pathology had the frontal variant of AD. This group has been defined before, and their neuropsychiatric profile has been described as well, but not in a pathologically confirmed sample [34]. Future research with the NACC data set or in prospectively studied groups might distinguish different neuropsychiatric profiles in more typical AD and pathologically defined groups of frontal variant $\mathrm{AD}$ and bvFTD patients.

Perhaps most interestingly, we found differences between tau-positive and tau-negative bvFTD groups. Tau-negative patients were, as a group, more likely to have delusions, depression, and changes in appetite and eating. There is little literature for comparison, although patients bearing the newly characterized C90RF72 mutation tend to have more psychotic behavior and possibly less appetitive changes [30]. Future research with scales probing the full range of neuropsychiatric symptoms in bvFTD such as the FBI may be more informative in determining the predictive relationship between neuropsychiatric symptoms and pathology. However, the information from this study may be pertinent to clinical diagnosis and will be informative to these future studies.

A limitation of this study includes the fact that the NACC database does not supply neuroanatomical details about the distribution of pathological findings. This will be important since it is likely that the specific networks disrupted, rather than the particular cellular changes, lead to particular behaviors. The relationship of particular neuropsychiatric changes and specific neuroanatomically localized pathologic burden has been shown in $\mathrm{AD}$, where postmortem neurofibrillary tangle counts in the left and right orbitofrontal and in the left anterior cingulate were found to be correlated with agitation, while load in the left anterior cingulate alone correlated with chronic apathy [35]. Furthermore, specific networks or cell types may have particular vulnerability to certain pathologies [36]. As more advanced neuroimaging techniques are being applied to the problem of predicting pathology and the neuroanatomical signature of the various pathologies is becoming more precise [37], these applications may further reveal the underlying networks involved in the various neuropsychiatric symptoms.

Another limitation, related to the retrospective nature of the database, involves the nonavailability of newer staining techniques for the analysis of the neuropathological changes. This is particularly true for the different forms of tau-negative inclusions (that currently number at least 7: 4 TDP-43 and 3 FUS varieties), which have been much better characterized over the last 5 years and which show fairly specific association with genetic variants and certain phenotypic presentations [2].

While this study concentrated on neuropsychiatric symptoms in relative isolation, phenotypes of FTLD pathology are more likely to be defined by combinations of neuropsychiatric, cognitive, and motor symptoms. In a pathologically confirmed series, Hodges et al. [38] found that behavioral symptoms were associated with a range of pathologies, while other clinical phenotypes had relatively uniform underlying pathologies: motor neuron disease predicted tau-negative inclusions, parkinsonism and apraxia predicted corticobasal (tau-positive) pathology, and nonfluent aphasia predicted tau-positive Pick bodies. Since motor and behavioral symptoms usually co-occur in the same patients, clustering of signs and behavioral changes should be explored further. The current study concentrated on bvFTD; however, neuropsychiatric symptoms occur in the language variant [39] and this is also pertinent to explore. 
Dementia

and Geriatric

Cognitive Disorders \begin{tabular}{l|l}
\hline Dement Geriatr Cogn Disord 2014;37:104-112 \\
\hline DOI: $\underline{10.1159 / 000354368}$ & $\begin{array}{l}\text { @ 2013 S. Karger AG, Basel } \\
\text { www.karger.com/dem }\end{array}$ \\
\hline
\end{tabular}

Léger and Banks: Neuropsychiatric Symptom Profile Differs Based on Pathology in

Patients with Clinically Diagnosed Behavioral Variant Frontotemporal Dementia

Shortly after the submission of this paper, a similar study of the NACC data was published by Mendez et al. [40]. Their study was based on a similar data subset. The data lock used in their study ended in July 2011, 8 months before the data lock in our study (March 2012), reducing the total number of subjects available in their study. The statistical analyses were equivalent. As found in this study, slightly more than $20 \%$ of patients with clinically diagnosed bvFTD were found to have AD pathology. The authors were able to show that patients with bvFTD-AD (i.e. with AD pathology) in comparison to patients with bvFTD-FTLD (i.e. with FTLD pathology) were more likely to have memory loss and less likely to show judgment/ problem solving deficits or personality change as first symptoms. Personality change remained the only significant 'current' symptom difference (i.e. from the most recent visit as opposed to the initial visit). Also similar to our findings, bvFTD-AD patients were more likely to show hallucinations, delusions, or agitation on the NPI-Q. When comparing the tau-negative and tau-positive pathologies, they found that only personality change was significantly more frequent in tau-positive patients; additionally, they found no statistical differences in NPI-Q question endorsement. In contrast, we found that tau-negative patients were more likely to demonstrate depression, delusions, and changes in appetite and eating. This discrepancy is most likely explained by the inclusion of a larger number of subjects in our study.

Future studies may use prospective techniques to predict pathology based on symptoms, including neuropsychiatric manifestations. Novel PET ligands and genetics will soon make 'pathological diagnosis' possible during life, allowing for the in vivo correlation of clinical phenotypes with underlying pathology. To that end, the findings of this study represent a first small step in this direction.

\section{Acknowledgments}

We would like to thank Dr. Jeffery Cummings for his invaluable comments on the first draft of the manuscript. Creation and maintenance of the NACC database is supported by NACC grant No. U01 AG016976.

\section{References}

- 1 Neary D, Snowden J, Gustafson L, Passant U, Stuss D, Black S, et al: Frontotemporal lobar degeneration: a consensus on clinical diagnostic criteria. Neurology 1998;51:1546-1554.

- 2 Rademakers R, Neumann M, Mackenzie IR: Advances in understanding the molecular basis of frontotemporal dementia. Nat Rev Neurol 2012;8:423-434.

3 Halliday G, Bigio EH, Cairns NJ, Neumann M, Mackenzie IR, Mann DM: Mechanisms of disease in frontotemporal lobar degeneration: gain of function versus loss of function effects. Acta Neuropathol 2012;124:373382.

4 Gorno-Tempini ML, Hillis AE, Weintraub S, Kertesz A, Mendez M, Cappa SF, et al: Classification of primary progressive aphasia and its variants. Neurology 2011;76:1006-1014.

- 5 Chow TW, Alobaidy AA: Incorporating new diagnostic schemas, genetics, and proteinopathy into the evaluation of frontotemporal degeneration. Continuum (Minneap Minn) 2013;19:438-456.

- 6 Bigni B, Premi E, Pilotto A, Padovani A, Borroni B: Disease-modifying therapies in frontotemporal lobar degeneration. Curr Med Chem 2012;19:1008-1020.

- 7 Rascovsky K, Hodges JR, Knopman D, Mendez MF, Kramer JH, Neuhaus J, et al: Sensitivity of revised diagnostic criteria for the behavioural variant of frontotemporal dementia. Brain 2011;134:2456-2477.

- 8 Cummings JL, Mega M, Gray K, Rosenberg-Thompson S, Carusi D, Gornbein J: The Neuropsychiatric Inventory: comprehensive assessment of psychopathology in dementia. Neurology 1994;44:2308-2314.

- 9 Barber R, Snowden J, Craufurd D: Frontotemporal dementia and Alzheimer's disease: retrospective differentiation using information from informants. J Neurol Neurosurg Psychiatry 1995;59:61-70.

10 Kertesz A, Davidson W, Fox H: Frontal behavioral inventory: diagnostic criteria for frontal lobe dementia. Can J Neurol Sci 1997;24:29-36.

11 Mendez MF, Perryman KM, Miller BL, Cummings JL: Behavioral differences between frontotemporal dementia and Alzheimers disease: a comparison on the BEHAVE-AD rating scale. Int Psychogeriatr 1998;10:155-162.

12 Lebert F, Pasquier F, Souliez L, Petit H: Frontotemporal behavioral scale. Alzheimer Dis Assoc Disord 1998; 12:335-339. 
Léger and Banks: Neuropsychiatric Symptom Profile Differs Based on Pathology in

Patients with Clinically Diagnosed Behavioral Variant Frontotemporal Dementia

13 Bozeat S, Gregory CA, Ralph MA, Hodges JR: Which neuropsychiatric and behavioural features distinguish frontal and temporal variants of frontotemporal dementia from Alzheimer's disease? J Neurol Neurosurg Psychiatry 2000;69:178-186.

14 Bathgate D, Snowden JS, Varma A, Blackshaw A, Neary D: Behaviour in frontotemporal dementia, Alzheimer's disease and vascular dementia. Acta Neurol Scand 2001;103:367-378.

$\checkmark 15$ De Deyn PP, Engelborghs S, Saerens J, Goeman J, Mariën P, Maertens K, et al: The Middelheim Frontality Score: a behavioural assessment scale that discriminates frontotemporal dementia from Alzheimer's disease. Int J Geriatr Psychiatry 2004;20:70-79.

16 Levy ML, Miller BL, Cummings JL, Fairbanks LA, Craig A: Alzheimer disease and frontotemporal dementias: behavioral distinctions. Arch Neurol 1996;53:687-690.

$\checkmark 17$ Rozzini L, Lussignoli G, Padovani A, Bianchetti A, Trabucchi M: Alzheimer disease and frontotemporal dementia. Arch Neurol 1997;54:350.

18 Hirono N, Mori E, Tanimukai S, Kazui H, Hashimoto M, Hanihara T, Imamura T: Distinctive neurobehavioral features among neurodegenerative dementias. J Neuropsychiatry Clin Neurosci 1999;11:498-503.

19 Kaufer DI, Cummings JL, Ketchel P, Smith V, MacMillan A, Shelley T, et al: Validation of the NPI-Q, a brief clinical form of the Neuropsychiatric Inventory. J Neuropsychiatry Clin Neurosci 2000;12:233-239.

20 Kertesz A, Nadkarni N, Davidson W, Thomas AW: The Frontal Behavioral Inventory in the differential diagnosis of frontotemporal dementia. J Int Neuropsychol Soc 2000;6:460-468.

-21 Marczinski CA, Davidson W, Kertesz A: A longitudinal study of behavior in frontotemporal dementia and primary progressive aphasia. Cogn Behav Neurol 2004;17:185-190.

22 Blair M, Kertesz A, Davis-Faroque N, Hsiung GY, Black SE, Bouchard RW, et al: Behavioural measures in frontotemporal lobar dementia and other dementias: the utility of the frontal behavioural inventory and the neuropsychiatric inventory in a national cohort study. Dement Geriatr Cogn Disord 2007;23:406-415.

-23 Clinical and neuropathological criteria for frontotemporal dementia. The Lund and Manchester Groups. J Neurol Neurosurg Psychiatry 1994;57:416-418.

-24 Rankin KP, Kramer JH, Mychack P, Miller BL: Double dissociation of social functioning in frontotemporal dementia. Neurology 2003;60:266-271.

25 Nagahama Y, Okina T, Suzuki N, Matsuda M: The Cambridge Behavioral Inventory: validation and application in a memory clinic. J Geriatr Psychiatry Neurol 2006;19:220-225.

26 Liscic RM, Storandt M, Cairns NJ, Morris JC: Clinical and psychometric distinction of frontotemporal and Alzheimer dementias. Arch Neurol 2007;64:535-540.

-27 Forman MS, Farmer J, Johnson JK, Clark CM, Arnold SE, Coslett HB, et al: Frontotemporal dementia: clinicopathological correlations. Ann Neurol 2006;59:952-962.

28 Kulisevsky J, Roldan E: Hallucinations and sleep disturbances in Parkinson's disease. Neurology 2004;63:S28S30.

29 Compta Y, Martí MJ, Rey MJ, Ezquerra M: Parkinsonism, dysautonomia, REM behaviour disorder and visual hallucinations mimicking synucleinopathy in a patient with progressive supranuclear palsy. J Neurol Neurosurg Psychiatry 2009;80:578-579.

-30 Snowden JS, Rollinson S, Thompson JC, Harris JM, Stopford CL, Richardson AM, et al: Distinct clinical and pathological characteristics of frontotemporal dementia associated with C90RF72 mutations. Brain 2012;135: 693-708.

-31 Josephs KA, Hodges JR, Snowden JS, Mackenzie IR, Neumann M, Mann DM, Dickson DW: Neuropathological background of phenotypical variability in frontotemporal dementia. Acta Neuropathol 2011;122:137-153.

\$2 Whitwell JL, Josephs KA: Neuroimaging in frontotemporal lobar degeneration - predicting molecular pathology. Nat Rev Neurol 2011;8:131-142.

-33 Urwin H, Josephs KA, Rohrer JD, Mackenzie IR, Neumann M, Authier A, et al: FUS pathology defines the majority of tau- and TDP-43-negative frontotemporal lobar degeneration. Acta Neuropathol 2010;120:33-41.

-34 Woodward M, Jacova C, Black SE, Kertesz A, Mackenzie IR, Feldman H, et al: Differentiating the frontal variant of Alzheimer's disease. Int J Geriatr Psychiatry 2010;25:732-738.

-35 Tekin S, Mega MS, Masterman DM, Chow T, Garakian J, Vinters HV, et al: Orbitofrontal and anterior cingulate cortex neurofibrillary tangle burden is associated with agitation in Alzheimer disease. Ann Neurol 2001;49: 355-361.

-36 Kim EJ, Sidhu M, Gaus SE, Huang EJ, Hof PR, Miller BL, et al: Selective frontoinsular von Economo neuron and fork cell loss in early behavioral variant frontotemporal dementia. Cereb Cortex 2012;22:251-259.

37 Whitwell JL, Jack CR, Parisi JE, Knopman DS, Boeve BF, Petersen RC, et al: Imaging signatures of molecular pathology in behavioral variant frontotemporal dementia. J Mol Neurosci 2011;45:372-378.

-38 Hodges JR, Davies RR, Xuereb JH, Casey B, Broe M, Bak TH, et al: Clinicopathological correlates in frontotemporal dementia. Ann Neurol 2004;56:399-406.

-39 Banks SJ, Weintraub S: Neuropsychiatric symptoms in behavioral variant frontotemporal dementia and primary progressive aphasia. J Geriatr Psychiatry Neurol 2008;21:133-141.

40 Mendez MF, Joshi A, Tassniyom K, Teng E, Shapira JS: Clinicopathologic differences among patients with behavioral variant frontotemporal dementia. Neurology 2013;80:561-568. 